\title{
Assessing the feasibility of OpenCL CPU implementations for agent-based simulations
}

\author{
Nuno Fachada \\ Institute for Systems and Robotics (ISR/IST), LARSyS, \\ Instituto Superior Técnico \\ Av. Rovisco Pais, 1 \\ Lisboa 1049-001, Portugal \\ nfachada@laseeb.org
}

\begin{abstract}
Agent-based modeling (ABM) is a bottom-up modeling approach, where each entity of the system being modeled is uniquely represented as a self-determining agent. Large scale emergent behavior in ABMs is population sensitive. As such, it is advisable that the number of agents in a simulation is able to reflect the reality of the system being modeled. This means that in domains such as social modeling, ecology, and biology, systems can contain millions or billions of individuals. Such large scale simulations are only feasible in non-distributed scenarios when the computational power of commodity processors, such as GPUs and multi-core CPUs, is fully exploited. In this paper we evaluate the feasibility of using CPUoriented OpenCL for high-performance simulations of agent-based models. We compare a CPU-oriented OpenCL implementation of a reference ABM against a parallel Java version of the same model. We show that there are considerable gains in using CPU-based OpenCL for developing and implementing ABMs, with speedups up to $10 \times$ over the parallel Java version on a 10-core hyper-threaded CPU.
\end{abstract}

\section{CCS CONCEPTS}

•Computing methodologies $\rightarrow$ Shared memory algorithms; Agent / discrete models; Massively parallel and high-performance simulations; Parallel programming languages; Model verification and validation; Artificial life;

\section{KEYWORDS}

Parallel simulation, Agent-based model, PPHPC, OpenCL, cf4ocl

\section{ACM Reference format:}

Nuno Fachada and Agostinho C. Rosa. 2017. Assessing the feasibility of OpenCL CPU implementations for agent-based simulations. In Proceedings of IWOCL '17, Toronto, Canada, May 16-18, 2017, 10 pages.

DOI: http://dx.doi.org/10.1145/3078155.3078174

Permission to make digital or hard copies of all or part of this work for personal or classroom use is granted without fee provided that copies are not made or distributed for profit or commercial advantage and that copies bear this notice and the full citation on the first page. Copyrights for components of this work owned by others than the author(s) must be honored. Abstracting with credit is permitted. To copy otherwise, or republish, to post on servers or to redistribute to lists, requires prior specific permission and/or a fee. Request permissions from permissions@acm.org.

IWOCL '17, Toronto, Canada

(c) 2017 Copyright held by the owner/author(s). Publication rights licensed to ACM 978-1-4503-5214-7/17/05 ..\$15.00

DOI: http://dx.doi.org/10.1145/3078155.3078174

\author{
Agostinho C. Rosa \\ Institute for Systems and Robotics (ISR/IST), LARSyS, \\ Instituto Superior Técnico \\ Av. Rovisco Pais, 1 \\ Lisboa 1049-001, Portugal \\ acrosa@laseeb.org
}

\section{INTRODUCTION}

Agent-based modeling (ABM) is a bottom-up modeling approach, where each entity of the system being modeled is uniquely represented as a self-determining agent. When prompted to act, each agent examines its current situation (e.g. what resources are available, what other agents are in the vicinity), and acts accordingly, based on a set of rules (e.g., if-then-else rules, differential equations, neural networks). These rules incorporate knowledge or theories about the respective low-level components. The global behavior of the system then emerges from the simple, self-organized local relationships between the agents [13]. As such, ABM is a useful tool in simulating and exploring systems that can be modeled in terms of interactions between individual agents, for example, biological cell cultures, ants foraging for food or humans in a crowd.

Spatial agent-based models (SABMs) are a subset of ABMs in which a spatial topology determines how agents interact [56]. For example, an agent may be limited to interact with agents located within a specific radius, or may only move to a near physical or geographical location [45]. SABMs have been extensively used to study a range of phenomena in the biological and social sciences $[36,56]$.

Large scale emergent behavior in ABMs is population sensitive. As such, it is advisable that the number of agents in a simulation is able to reflect the reality of the system being modeled [35, 38, 44]. Otherwise, accurate simulation behavior may not be attainable, and model validation becomes difficult $[31,35]$. This means that in domains such as social modeling, ecology, and biology, systems can contain millions or billions of individuals [9, 10, 38, 53]. Consequently, simulating realistic models will involve as much agents being processed per time step [9]. Such large scale simulations generate a very high demand for computing power [30] and are impractical on typical ABM frameworks such as NetLogo [69] or Repast Simphony [49], which run on a single CPU core or compute unit $[9,10]$. Considering that commodity processors, such as GPUs and multi-core CPUs, are nowadays composed of several compute units, a natural solution to reach acceptable scalability in ABMs consists of decomposing models such that each component can be autonomously processed by a logical processor $\left(\mathrm{LP}^{1}\right)$ in a concurrent manner [5, 31, 56, 60-62, 66]. However, in order to minimize communication between LPs and obtain optimal performance, model partitioning should guarantee that individual model components are as independent as possible.

\footnotetext{
${ }^{1}$ In shared memory architectures, LPs are usually represented by threads, which communicate via synchronized access to shared variables. In distributed memory scenarios, LPs are commonly represented by processes, which communicate via message passing.
} 
In this paper we evaluate the feasibility of using CPU-oriented OpenCL for high-performance simulations of agent-based models. OpenCL is an open standard for general-purpose parallel programming across CPUs, GPUs, FPGAs and other processors, giving software developers portable and efficient access to the power of these heterogeneous processing platforms [34, 57]. OpenCL implementations are provided by a large and increasing number of $\mathrm{CPU}$, GPU and FPGA vendors ${ }^{2}$. We test a CPU-oriented OpenCL implementation of the reference PPHPC ABM [16] over two proprietary OpenCL CPU runtimes, provided by Intel and AMD, respectively. We compare this implementation against a parallel Java version of the same model [20], both in terms of performance and statistical accuracy. Leveraging on the fact that the PPHPC model captures important characteristics of SABMs, such as agent movement and local agent interactions, several conclusions on SABM parallelization techniques using OpenCL are drawn.

The rest of the paper is organized as follows. First, in Section 2, previous work on SABM parallelization is presented and the associated issues are discussed. Next, Section 3, Methods, is composed of five parts: 1) an overview of the PPHPC model; 2) a discussion on how to generically parallelize it; 3) a description of the model's parallel Java implementation; 4) a thorough presentation of the CPU-oriented OpenCL implementation of PPHPC; and, 5) a summary on how to compare the outputs collected from two model implementations in order to assert their statistical equivalence. Results, Section 4, show that there are considerable gains in using CPU-based OpenCL for developing and implementing ABMs, and at the same time guaranteeing simulation reproducibility and statistical accuracy with the original model. Section 5 provides some conclusion of what was accomplished in this paper, outlining directions for future work.

\section{BACKGROUND}

The distributed memory programming model is widely used for parallelizing ABMs $[4,5,30,55,56,62]$. This approach allows models to scale to thousands of cores, usually found in supercomputer-type setups or computing clusters. However, communication issues for larger models [56] and a more complex programming paradigm (when compared with multithreading on shared memory architectures) [41] often restrict this approach.

Recently, the trend has been on hybrid [1, 59], GPU $[3,44,54,68]$ and heterogeneous $[65,67]$ methods. While these approaches allow concrete gains in simulation performance on commodity hardware, they come with an increased cost in implementation time due to the considerably more complex programming models. Hybrid methods, combining distributed and shared memory programming models, require modelers to master both paradigms, as well as specific multi-level model decomposition. GPU architectures require the reformulation of ABMs in terms of stream SIMD computation and offer limited control flow constructs [44]. Heterogeneous methods, in which both CPU and GPU are utilized, require careful model decomposition so that components can be efficiently processed. Furthermore, costly data transfers between the two processors can only be avoided by using integrated GPUs, which are typically much less powerful than their discrete counterparts.

\footnotetext{
${ }^{2}$ https://www.khronos.org/conformance/adopters/conformant-products\#opencl
}

The computing potential of GPUs and the apparent massively parallel nature of SABMs make general-purpose GPU (GPGPU) implementations of such models an interesting proposal [52]. However, in order to express ABMs in terms of stream SIMD computation, each type of agent should be processed by the same code (single instruction) and multiple instances of each type of agent should exist in the simulation (multiple data) [32, 42]. Optimal performance requires that the processing of one agent is causally independent of the processing of any other agent in the same time step [9]. This can be difficult to achieve with ABMs, since the behavior of an agent is frequently associated with the dynamic environment and with the actions of other agents [42]. A common case in SABMs is that of mobile agents in a discrete grid, which poses several difficulties. If there is a unique location constraint (maximum of one agent per grid cell), the implementation must explicitly handle collisions which result from agents concurrently moving to the same location [44, 54]. However, the absence of this constrain creates a different problem. More specifically, agents need to determine the location of other agents in their neighborhood. The typical solution consists of having a sorted array of agents, with each grid cell holding a pointer to the first agent it contains. This solution requires a sorting step in each iteration, which can deteriorate performance $[2,12,14,28,51]$. The agent life-cycle is another difficult aspect of ABM on GPUs, requiring adequate memory management patterns, such as allocation and garbage collection, which are unavailable within GPGPU kernels [44]. For more complex models, with different agent types and dynamic environments which influence agent behavior, it will be difficult to find an efficient SIMD solution at all [42]. Having this into account, the use of SIMD architectures may lead to good results for simpler ABMs [42], such as cellular automata and homogeneous agent models $[4,24,54]$. A number of proposed GPGPU ABM frameworks rely on these assumptions or simplifications [4, 35, 44, 54].

Among the possible parallelization techniques, multithreading is arguably the simplest to implement $[41,66]$, with the added bonus of portability. For example, modern threading APIs, such as OpenMP [8] for C, C++ and Fortran or the Java concurrency API [25] greatly simplify multithreaded ABM implementations, and are available for a number of different shared memory CPU architectures and operating systems. For example, the ABM of opinion exchange presented by Gong et al. [27], implemented in $\mathrm{C}++$ and parallelized with OpenMP, was tested in a 32-core CPU (AMD Opteron), with impressive speedups. Java is also a common choice for parallelizing ABMs, as exemplified by Fachada et al. $[13,20]$ and Goldsby and Pancerella [26].

A few ABMs have also been developed in OpenCL in recent years. However, most have either been focused on GPGPU [6, 48] or implemented without apparent CPU-specific optimizations when also tested on CPUs [24, 37, 39, 40]. The work of Wang et al. [67] is an exception, leveraging OpenCL's versatility by targeting heterogeneous processors, i.e., CPUs with integrated GPUs. 


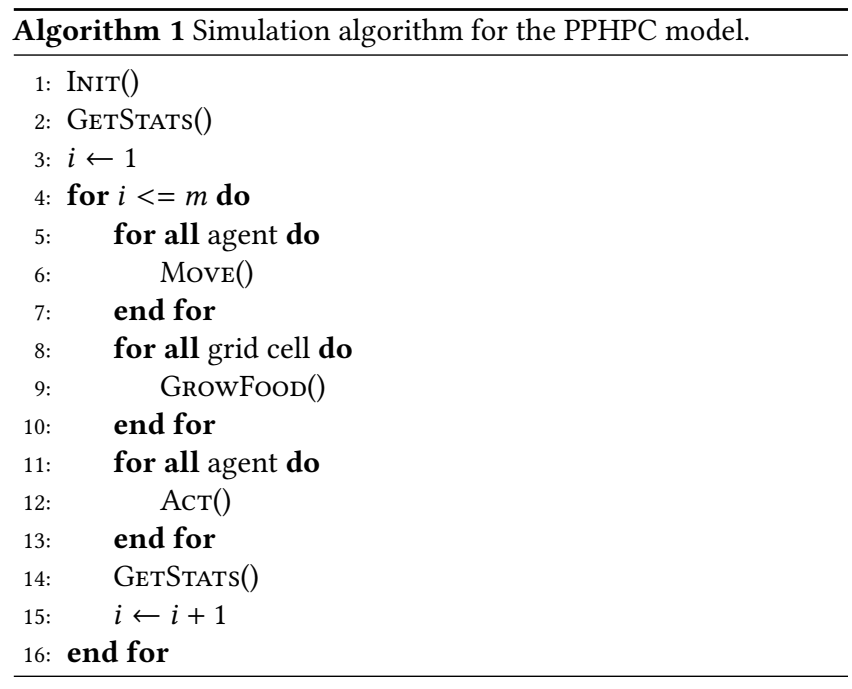

\section{METHODS}

\subsection{The PPHPC agent-based model}

The Predator-Prey for High-Performance Computing (PPHPC) model is a reference model for studying and evaluating SABM implementation strategies, capturing important characteristics such as agent movement and local agent interactions. It is used in this work as a test case for ABM parallelization using CPU-oriented OpenCL. The model is thoroughly described in reference [16] using the ODD protocol [29]. Here we present a summarized description of the model.

PPHPC is a predator-prey model composed of three entity classes: agents, grid cells and environment. Agents can be of type prey or predator. While prey consume passive cell-bound food, predators consume prey. Agents have an energy state variable, $E$, which increases when feeding and decreases when moving and reproducing. When energy reaches zero, the agent is removed from the simulation. Instances of the grid cell entity class are where agents act, namely where they try to feed and reproduce. Grid cells have a fixed grid position and contain only one resource, cell-bound food (grass), which can be consumed by prey, and is represented by the countdown state variable $C$. The $C$ state variable specifies the number of iterations left for the cell-bound food to become available. Food becomes available when $C=0$, and when a prey consumes it, $C$ is set to $c_{r}$ (an initial simulation parameter). The set of all grid cells forms the environment entity, a toroidal square grid where the simulation takes place. The environment is defined by its size and by the restart parameter, $c_{r}$. The temporal scale is represented by a positive integer $m$, which represents the number of iterations.

Simulations start with an initialization process, where a predetermined number of agents are randomly placed in the simulation environment. Cell-bound food is also initialized at this stage. After initialization, and to get the simulation state at iteration zero, outputs are collected. The scheduler then enters the main simulation loop, where each iteration is sub-divided into four steps: 1) agent movement; 2) food growth in grid cells; 3) agent actions; and, 4) gathering of simulation outputs. This process is summarized in Algorithm 1.
Six outputs are collected at each iteration $i$ : prey population $\left(P_{i}^{s}\right)$, predator population $\left(P_{i}^{w}\right)$, quantity of cell-bound food $\left(P_{i}^{c}\right)$, mean prey energy $\left(\bar{E}_{i}^{s}\right)$, mean predator energy $\left(\bar{E}_{i}^{w}\right)$ and mean value of the $C$ state variable in all grid cells $\left(\bar{C}_{i}\right)$.

Reference parameters for the PPHPC model are qualitatively separated into size-related and dynamics-related groups [16]. Although size-related parameters also influence model dynamics, this separation is useful for parameterizing simulations. Concerning size-related parameters, a base grid size of $100 \times 100$ is associated with 400 prey and 200 predators. Different grid sizes should have proportionally assigned agent population sizes, such that the initial agent density and the initial ratio between prey and predators remains constant. Thus, model size is the relation between grid size and initial agent population. For example, model size 400 corresponds to a grid size of $400 \times 400$ with 6400 prey and 3200 predators at iteration 0 .

For the dynamics-related parameters, two parameter sets, 1 and 2 , are proposed. The two parameterizations generate distinct dynamics, with the second set typically yielding more than twice the number of agents than the first during the course of a simulation. The combination of model size and parameter set will be referred as "size/set", e.g., 400/1 for model size 400 , parameter set 1 . The reference number of iterations, $m$, is 4000 , not counting with the initial simulation state at iteration 0 .

\subsection{A generic parallelization approach}

The components that constitute a SABM can be split among LPs according to two broad patterns: agent-parallel (AP) and environment-parallel (EP) [50]. Even though these patterns are not mutually exclusive, they constitute a convenient basis for evaluating SABM parallelization techniques. In the AP criterion each LP processes a set of agents, i.e., the model is divided at the agent-level, as shown in Figure 1a. Load balancing is simplified since agents, and the associated computational tasks, can be equally distributed among LPs [7, 50]. However, in a moving agents scenario, additional communication between LPs is required in order to guarantee that spatially localized agent interactions are processed in a consistent fashion, as co-location on a LP does not guarantee co-location in space [50]. Model partitioning occurs at the spatial environment level in the EP criterion, i.e., each LP is assigned a slice of the simulation space, together with the agents it contains [7], as shown in Figure 1b. As such, local agent interactions will mostly occur in the same LPs. Unfortunately, load balancing issues may occur if spatial agent density is mostly non-uniform. This typically occurs in models involving agent grouping phenomena such as flocking or chemotaxis [7,21, 26, 50].

As shown in Algorithm 2, the parallelization approach followed by the PPHPC implementations discussed here is essentially based on the EP pattern (cell initialization and main simulation loop), with the AP paradigm used only for agent initialization.

The Init () process of Algorithm 1 is represented in lines 1-3 of Algorithm 2 by the InitCells(), InitPrey() and InitPredators() subprocesses. The first is executed in EP fashion, since there are no dependencies on initializing the cells and setting the initial cell-bound food. The two agent initialization processes are executed in AP fashion, i.e., each LP manages the initialization and 


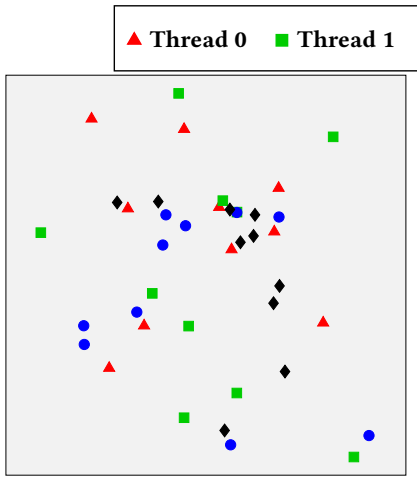

(a) Agent-parallel.

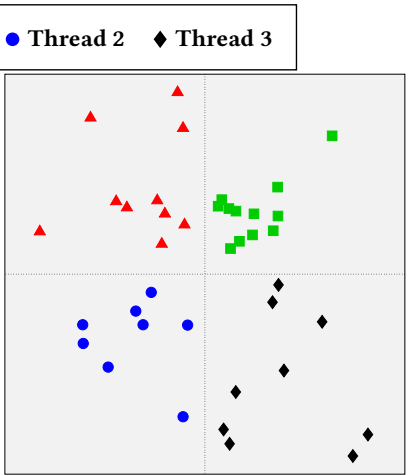

(b) Environment-parallel.

Figure 1: The SABM parallelization patterns.

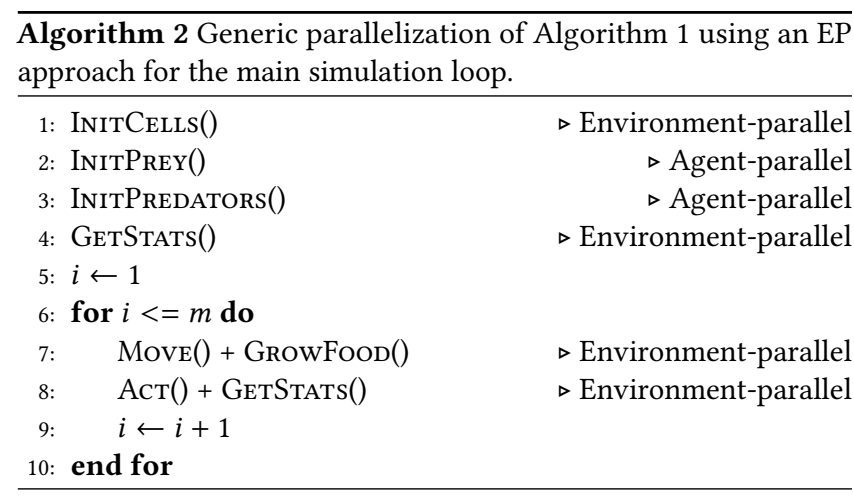

deployment of a number of agents, although, as we shall discuss, this is done differently in the Java and OpenCL implementations.

The GetStats() process (line 2 of Algorithm 1 and line 4 of Algorithm 2) collects the initial simulation output using an EP strategy, since at this stage cells are independent of each other.

Lines 5-10 of Algorithm 1, which include agent movement and growth of cell-bound food, are combined into a single EP loop (line 7 of Algorithm 2). This is possible because the Move() and GrowFood() processes are independent; i.e., the outcomes of either will only influence the Act () process, which occurs later. Basically, both Move() and GrowFood() are cell-wise independent and can be processed autonomously for each cell in an EP loop. When a cell is evaluated, agents located within are prompted to move, and then the cell is asked to execute its GrowFood() process. Care must be taken so that agents that already moved are not prompted to move again.

Finally, lines 11-14 of Algorithm 1, containing the Act() and GetStats() processes, are also condensed into one EP loop, as shown in line 8 of Algorithm 2. While agent actions and statistics gathering are not independent events (i.e., the former must occur before end-of-iteration data is collected), they are cell-wise independent. As described in Section 3.1, the actions of an agent are limited to the cell it occupies. Thus, after agent actions take place in a cell, end-of-iteration cell data can be collected.

\begin{tabular}{|c|c|c|}
\hline PS & Rep. & Description \\
\hline ST & Yes & Serial execution (no parallelization). \\
\hline EQ & No & Partition grid between LPs, cell-level sync. \\
\hline EX & Yes & $\begin{array}{l}\text { Same as previous, with ordered agent inser- } \\
\text { tion. }\end{array}$ \\
\hline ER & Yes & $\begin{array}{l}\text { LPs simultaneously process grid rows, sync. } \\
\text { at end of row. }\end{array}$ \\
\hline OD & No & $\begin{array}{l}\text { LPs continuously process blocks of grid cells } \\
\text { while they are available, cell-level sync. }\end{array}$ \\
\hline
\end{tabular}

Table 1: Parallelization strategies (PS) in the Java implementation. The second column (Rep.) indicates if simulations performed with the respective PS are numerically reproducible.

\subsection{A Java implementation}

Java is a well-known language within the ABM community, powering popular toolkits such as NetLogo [69], Repast Simphony [49] and MASON [43]. The Java implementation of PPHPC [20] is based on the concept of units of work, which are processed by one or more worker threads. The basic units of work are grid cells in the EP sections of Algorithm 2, and agents in the AP sections. This implementation was designed using a modular and scalable approach, following relatively high-level object-oriented programming concepts and design patterns [23]. However, care has been taken so that performance was not compromised. Consequently, the implementation has shown speedups up to $40 \times$ on a six-core hyper-threaded processor against a serial version implemented in NetLogo [20].

This implementation of PPHPC follows the Model-View-Controller design pattern. The Model component represents the actual ABM logic, and can be manipulated and observed using one or more views. Work factories, implementing the factory pattern, are responsible for creating and configuring the controller and work providers, which handle how units of work are processed. The controller spawns a specified number of worker threads, which in turn rely on the controller for synchronization. The work providers determine the amount of work each worker processes. Five work factories are provided, each implementing a specific parallelization strategy, as shown in Table 1.

The EX strategy is selected for this comparison, as it allows for numerically reproducible simulations while offering a level of performance close to that of the top-performing OD strategy [20]. The general idea of the EX parallelization strategy is that each worker always processes the same work. Work distribution is performed once at the beginning of the simulation by the associated work providers, and then the workers are always given the same exact units of work, i.e., they always process the same cells in the EP sections. Cell-level synchronization is required because more than one worker may potentially access the same cell at the same time for agent movement or initial agent placement purposes. Agents are orderly placed in cells, allowing them to posteriorly move or act in the same sequence, making numerically reproducible simulations possible. The maximum number of units of work to be processed by each worker, $n$, is given by 


$$
n=\lceil T / N\rceil
$$

where $T$ is the total number of units of work to be processed in a parallel work cycle, and $N$ is the number of worker threads. If $T$ is not equally divisible between the available workers, the last worker will process less work than the remaining workers, as shown in Eq. 2 ,

$$
n \cdot i \leq t_{i}<\min (n \cdot(i+1), T)
$$

where $i$ identifies the $i^{\text {th }}$ worker, and $t_{i}$ corresponds to the range of units of work which will be processed by the $i^{\text {th }}$ worker.

Parallel pseudo-random generation is carried out in the following manner. The $i^{\text {th }}$ worker thread obtains its own subsequence of a global random sequence by using a unique seed, $S_{i}$, through a random spacing approach [33]. Each seed $S_{i}$ is derived from the worker's unique identifier, $i$, and from a user specified global seed, $S$, according to Eq. 3,

$$
S_{i}= \begin{cases}S, & \text { if } i=0 \\ S \oplus \operatorname{SHA} 256(i), & \text { if } i>0\end{cases}
$$

where $\oplus$ is the bitwise XOR operator and SHA256() is the SHA-256 cryptographic hash function. Since each worker thread has its own PRNG subsequence, simulation reproducibility is guaranteed.

\subsection{A CPU-oriented OpenCL implementation}

The OpenCL CPU-oriented PPHPC realization uses OpenCL 1.2 kernels for maximum compatibility across vendor OpenCL CPU implementations. Additionally, host-side code was developed in C99, using the following libraries:

- GLib [63] - Used for command-line option parsing, error handling, pseudo-random number generation (host-side) and string manipulation.

- cf4ocl [18] - Simplifies OpenCL host-side programming and offers an integrated profiler.

- cl_ops [15] - Provides device-side pseudo-random number generators.

Due to the close-to-hardware nature of OpenCL, this PPHPC realization is implemented using a low-level approach, especially when compared with the Java version. First, the C99-based OpenCL kernel language does not allow for memory allocation inside kernels, meaning that: a) all the memory required for newly born agents must be host-side allocated upfront; and, b) the insertion of new agents in the simulation must be manually handled within the OpenCL kernels.

This PPHPC realization uses two data structures, more specifically two large arrays, in order to perform this management: 1) an array of grid cells; and, 2) an array of agents. While number of grid cells is fixed and known before the simulation begins, the same is not true for the number agents. As such, this array of agents should be made large enough to accommodate the maximum number of agents ever existing in the simulation.

Each element of the cells array is a structure containing two fields: 1) the grass countdown value, $C$; and, 2) the location (in the agents array) of the first agent in the cell. The array index implicitly identifies the cell position in the grid. In turn, each element of the agents array is a structure with the following fields: 1) the agent energy; 2) the agent type (predator or prey); and, 3) the index of the next agent in the same cell. The last agent in the cell points to a sentinel constant identifying the end of the list. As a consequence of this arrangement, agents in the same cell are scattered along the agents array, each agent holding a pointer to the next. These data structures are optimized for a CPU device. They would not be appropriate for GPU targets, due to the way the agents are scattered along the memory, and due to the use of the array of structures pattern (a structure of arrays is preferred for GPUs). Both approaches are not friendly towards coalesced memory accesses, one of the first optimizations to be considered for GPU targets.

Contrary to the Java version, the use of the EX strategy is not straightforward here. Following that approach, the insertion of an agent in a cell would require an atomic exchange between the first agent in the cell and the newly arrived agent. The latter would then become the first agent, pointing to the previously first agent - now the second agent in the cell. This would work, providing the equivalent of Java's EQ strategy, also sharing one of its caveats: it does not allow reproducible simulations. In order to obtain the equivalent of the EX strategy, new agents should be inserted in an orderly fashion. Since OpenCL does not provide Java-like explicitly synchronized blocks, this would have to be accomplished in one of two ways: a) via a spinlock implemented with atomics or a similar approach [58]; or, b) using an additional kernel, executed before agent movement and agent actions (i.e., before line 7 and before line 8 of Algorithm 2), to sort the agents within each cell according to a pseudo-random, but deterministic order. The former approach is somewhat against the spirit of OpenCL, is prone to deadlocks and may not be portable. The latter method may incur in severe computational overhead.

Given these considerations, we find that the ER (Equal with Row synchronization) parallelization strategy is a better fit to the OpenCL workflow. In the ER strategy, each worker serially processes rows of the simulation grid, leaving a distance of at least three rows (including the row to be processed) to the next worker (see Figure 2). More generally, this distance is given by

$$
d_{\min }=2 r+1
$$

where $r$ is the agent movement radius, which is 1 for the PPHPC model. This approach allows workers to run in parallel without cell-level synchronization, since they synchronize at the end of each row at the host level (i.e., between kernel invocations), as shown in Figure 2. Thus, agents always move to neighboring cells in the same order, making simulations reproducible.

An initial estimate of the number of rows to be processed by each worker, $\Delta y$, is given by

$$
\Delta y=\left\lfloor\frac{y_{\text {env }}}{N}\right\rfloor
$$

where $y_{\mathrm{env}}$ is the total number of rows in the simulation grid. This estimate can be incremented if: 1) the number of rows is not equally divisible by the number of workers; and, 2) after incrementing it, there are enough rows for the last worker to process. This is shown in Eq. 6, where $\Delta y_{f}$ is the final number of rows per worker: 


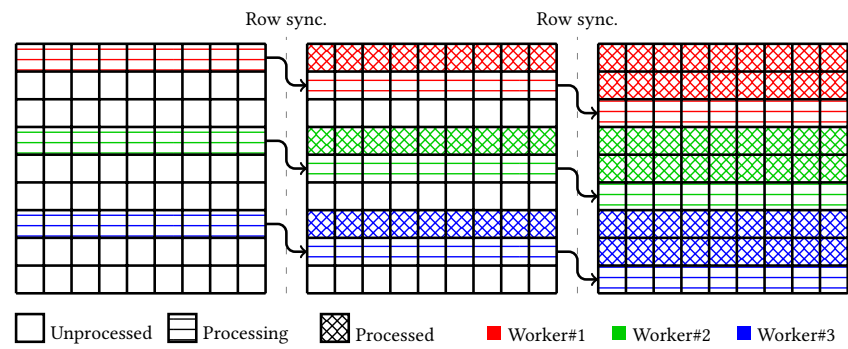

Figure 2: Equal with Row synchronization (ER): example of three workers processing nine rows of the simulation grid in parallel.

$\Delta y_{f}= \begin{cases}\Delta y+1, & \text { if } y_{\mathrm{env}} \bmod N>0 \wedge \\ & (N-1) \cdot(\Delta y+1) \leq y_{\mathrm{env}}-d_{\mathrm{min}} \\ \Delta y, & \text { otherwise }\end{cases}$

All workers, except possibly the last one, will process $n$ cells, according to Eq. 7. The exact number of cells that the $i^{\text {th }}$ worker will process are given in Eq. 8. Note that any adjustment due to the number of rows not being exactly divisible by the number of workers is performed on the last worker.

$$
\begin{gathered}
n=x_{\mathrm{env}} \cdot \Delta y_{f} \\
n \cdot i \leq t_{i}<t_{f}, \text { where } t_{f}= \begin{cases}n \cdot(i+1), & \text { if } i<N-1 \\
x_{\mathrm{env}} \times y_{\mathrm{env}}, & \text { if } i=N-1\end{cases}
\end{gathered}
$$

The OpenCL CPU implementation uses three kernels, init(), step1() and step2(), as shown in Algorithm 3. The ER strategy is implemented by kernels step1() and step2() in the main simulation loop, lines 4-13 of Algorithm 3. Each time these kernels are invoked, they process a row of the simulation environment, in accordance with the ER strategy. However, during the initialization stage, performed by the init() kernel (line 1 of Algorithm 3), a different parallelization approach, similar to Java's EX strategy, is used. The init() kernel implements the functionality of lines 1-4 of Algorithm 2, initializing grid cells, prey agents and predator agents, and collecting statistics for iteration 0. Each worker executing the init() kernel is assigned part of the environment grid. From here, workers initialize grid cells in EP fashion, and then proceed to initialize the agents. As in the case of cells, each worker initializes a similar amount of agents. However, in order to avoid conflicts, workers only insert agents in the cells assigned to them. This dispenses the use of atomics and ensures agents are deterministically placed in cells. In practice this goes beyond the PPHPC model specification, since it forces an even distribution of agents along the vertical axis of the environment grid at the beginning of the simulation.

Continuing to the main simulation loop kernels, step1() performs agent movement and grows cell food (line 7 of Algorithm 2), and step2() performs agent actions and gathering of end-of-iteration statistics (line 8 of Algorithm 2). In both cases, care is taken so that: a) agents that already moved/acted are not prompted to

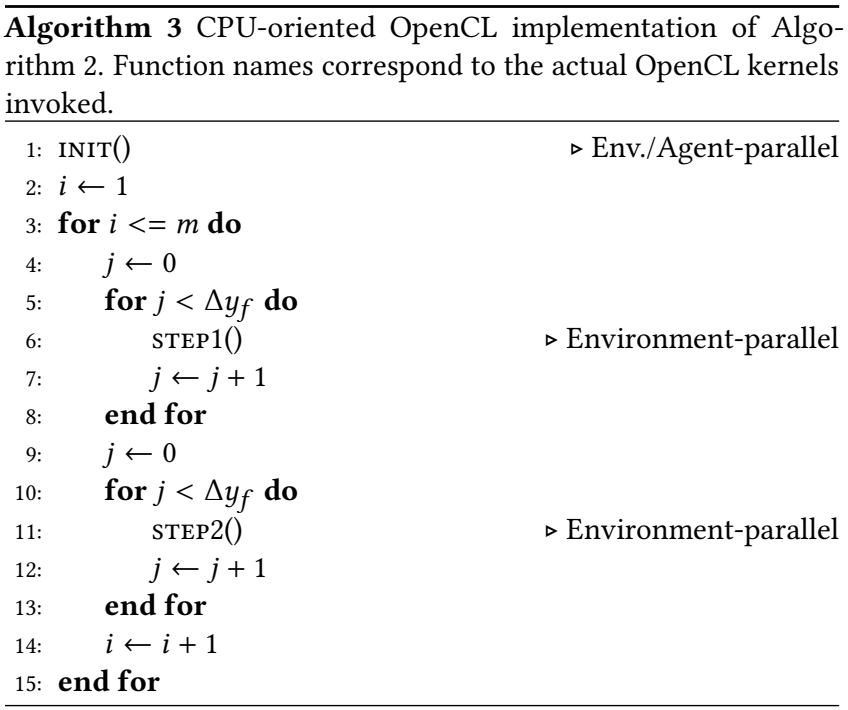

do so again; and, b) agents with zero energy or killed by predators are immediately removed from the simulation. Within work-items, i.e., OpenCL threads, agents are processed serially, maintaining simulation consistency.

Allocation of newly born agents in step2() is performed with an atomic compare-exchange instruction. A position in the agents array is randomly obtained, with two possible outcomes: 1) the position is empty, in which case it is atomically set as occupied; or, 2) the position is not empty, in which case random allocation is attempted again, up to a predefined, user-controllable maximum number of times. Only when allocation is successful are the state variables of the new agent written to the selected position. Although not deterministic, the positions of new agents are nonetheless irrelevant for simulation reproducibility.

The GetStats() process, executed in the step2() kernel, is performed by atomically updating a global array of structures. While this approach is not scalable for GPU targets - which favor reduction for this type of operation - it does not cause visible performance degradation in the CPU case, mostly likely due to the limited number of work-items executing in parallel.

Similar to the Java implementation, individual OpenCL workitems have their own subsequence of a global random sequence obtained via a random spacing approach. However, in this case the initial PRNG state for each work-item is determined serially at host-side with the Mersenne Twister PRNG [46] - in turn seeded with a user-specified value. Work-items then use and update their exclusive PRNG states with one of the PRNG algorithms provided by the cl_ops library, specifiable at run time.

\subsection{Statistical equivalence of model implementations}

The parallelization of ABMs is of no use if the parallel implementations do not produce statistically equivalent results with respect to the original serial version. It is very easy to inadvertently introduce changes which modify the model dynamic. This is a more complex type of model replication, which in itself is not a straightforward 
process $[11,70]$. ABMs are very sensitive to implementation details: the impact that seemingly unimportant aspects such as data structures, algorithms, discrete time representation, floating point arithmetic or order of events can have on results is tremendous $[47,70]$. The situation becomes more difficult with model parallelization, which by definition requires considerable changes in many of these aspects. Parry and Bithell [50] provide an informative account in which they were unable to successfully replicate a serial model when converting it to a parallel one.

In order to guarantee that the Java and OpenCL implementations display statistically indistinguishable behavior, we compare their output using a technique described in reference [19]. This model comparison method uses principal component analysis to convert simulation output into a set of linearly uncorrelated statistical measures, analyzable in a consistent, model-independent fashion. It is appropriate for ascertaining distributional equivalence of two or more model implementations, automatically selecting output features that best explain implementation differences.

\section{RESULTS AND DISCUSSION}

\subsection{Experimental setup}

A total of 10 replications were performed with the following combination of parameters:

- Implementations:

- Java: 20 threads, Mersenne Twister PRNG

- OpenCL: 20 work-items/work-groups (1 work-item per work-group), MWC64X PRNG [64]

- Parameter sets: 1,2

- Model sizes: 400, 800, 1600, 3200, 6400

For each combination of parameters, the 10 replications were performed with distinct PRNG seeds. These replications are the basis for both the performance and statistical analysis performed in the next sections. All performance results are based on the mean run time of the 10 replications. Replications were performed in "headless" mode for both implementations, i.e., without any graphical component. All replications were performed on a machine with the following hardware and software configuration:

- Intel Xeon CPU E5-2650 v3 @ 2.30GHz (ten cores, two logical processors per core), 64GB RAM

- Ubuntu 16.04.1 LTS, OpenJDK Java 1.8.0, Intel OpenCL CPU Runtime 16.1.1, AMD APP SDK 3.0

The source code of both PPHPC implementations is available at https://github.com/fakenmc/pphpc. The data produced by this computational experiment, as well as the scripts used to set up and analyze the experiment, are available at https://zenodo.org/record/ 293014.

\subsection{Performance comparison}

Figure 3 shows how the Java and OpenCL implementations scale for increasingly larger model sizes. Speedups of the OpenCL implementation (on Intel and AMD runtimes) against the Java implementation for the tested model sizes are displayed in Figure 4. Table 2 provides more detailed data, including simulation times and relative standard deviations. These figures and table were generated with PerfAndPubTools [17].

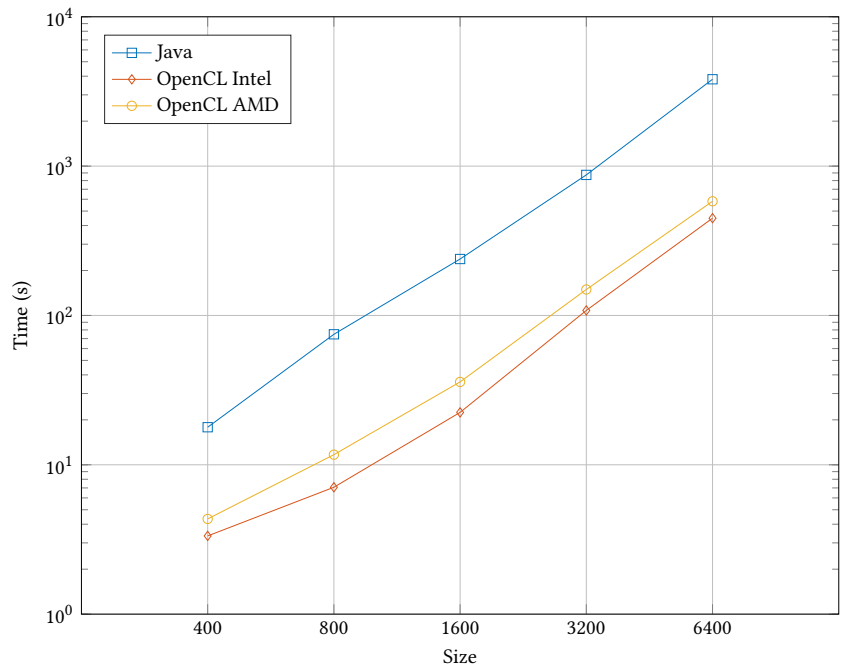

(a) Parameter set 1 .

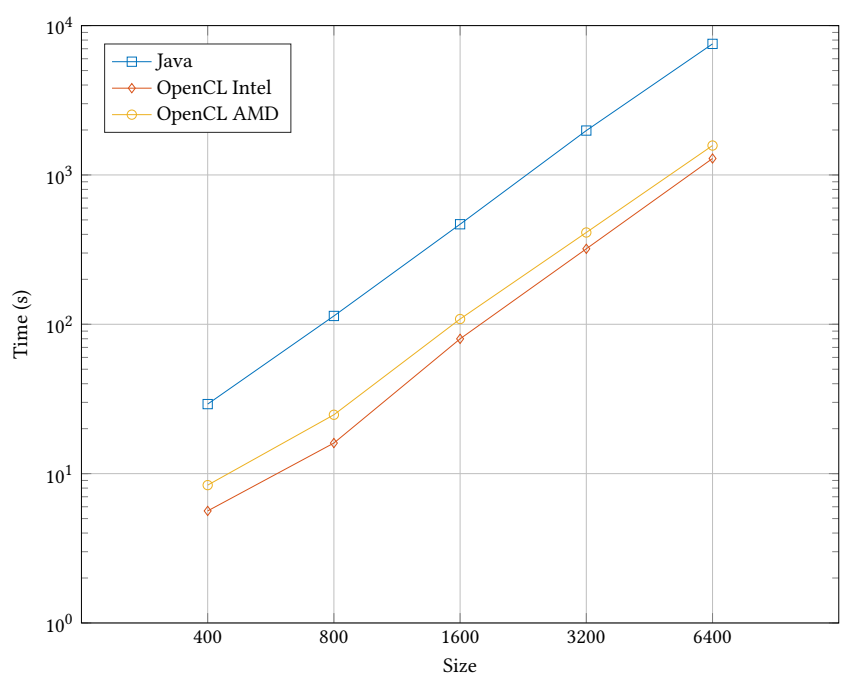

(b) Parameter set 2.

Figure 3: Scalability of the Java and OpenCL implementations for increasing model sizes.

With respect to the scalability of the several versions, it is clear the OpenCL implementation, on both Intel and AMD runtimes, is consistently more performant than the parallel Java realization. In other words, there is no noticeable relative approximation between OpenCL and Java for increasing model sizes. Interestingly, and while the Intel runtime is more efficient than AMD's for all tested size/parameter combinations, their relative performance differences seem to decrease for larger sizes.

Concerning speedups, the OpenCL implementation running on the Intel runtime is between $5 \times$ and $10 \times$ faster than the Java realization for parameter set 1 , stabilizing at $8 \times$ for the larger sizes. A similar speedup pattern is observed when running the OpenCL 


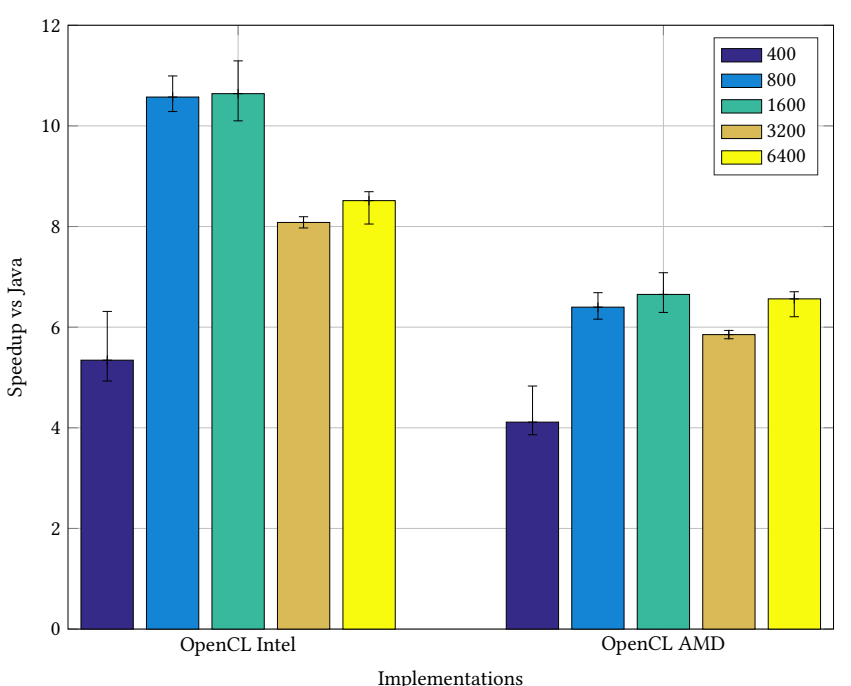

(a) Parameter set 1.

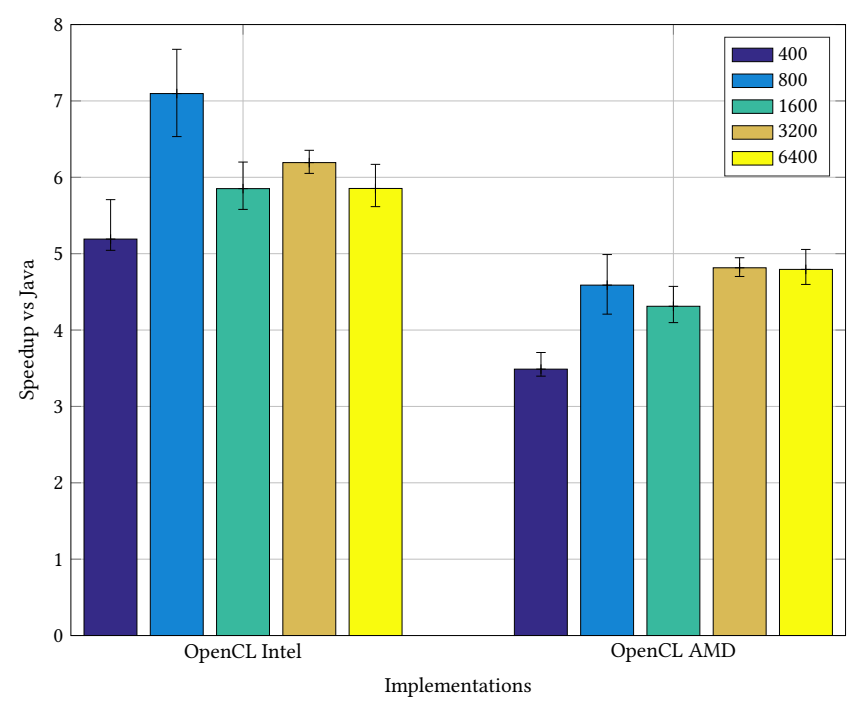

(b) Parameter set 2.

Figure 4: Speedups of the OpenCL implementation (on Intel and AMD runtimes) against the Java implementation for the tested model sizes. Error bars show the maximum and minimum speedups.

version on the AMD runtime, although the effective speedups are only $60 \%-75 \%$ of those verified on the Intel driver.

The speedups for second parameter set have less variation. For the OpenCL simulations performed with the Intel runtime, speedups over the Java implementation vary between $5 \times$ and $7 \times$. The AMD replications follow the trend observed for parameter set 1 , displaying a similar pattern to the Intel runs, with smaller speedups between $65 \%$ and $80 \%$ of Intel's runs. Thus, not only are the observed speedups more stable for the second parameter set, but the performance gap between the Intel and AMD runtimes is smaller.

\begin{tabular}{|c|c|c|c|c|c|c|c|}
\hline \multirow{2}{*}{ Impl. } & \multirow{2}{*}{ Size } & \multicolumn{3}{|c|}{ Param. set 1} & \multicolumn{3}{|c|}{ Param. set 2} \\
\hline & & $\bar{t}(\mathrm{~s})$ & $s(\%)$ & $S_{p}^{\text {Java }}$ & $\bar{t}(\mathrm{~s})$ & $s(\%)$ & $S_{p}^{\text {Java }}$ \\
\hline \multirow{5}{*}{ Java } & 400 & 17.85 & 8.4 & 1.00 & 29.21 & 2.19 & 1.00 \\
\hline & 800 & 74.79 & 1.54 & 1.00 & 113.70 & 5.23 & 1.00 \\
\hline & 1600 & 238.73 & 2.87 & 1.00 & 467.57 & 3.88 & 1.00 \\
\hline & 3200 & 873.28 & 0.91 & 1.00 & 1982.88 & 1.44 & 1.00 \\
\hline & 6400 & 3815.88 & 2.40 & 1.00 & 7545.00 & 3.55 & 1.00 \\
\hline \multirow{5}{*}{ CL-I } & 400 & 3.34 & 1.33 & 5.34 & 5.63 & 1.88 & 5.19 \\
\hline & 800 & 7.07 & 0.65 & 10.57 & 16.02 & 0.55 & 7.10 \\
\hline & 1600 & 22.44 & 0.38 & 10.64 & 79.90 & 0.14 & 5.85 \\
\hline & 3200 & 108.07 & 0.11 & 8.08 & 320.20 & 0.05 & 6.19 \\
\hline & 6400 & 448.16 & 0.07 & 8.51 & 1288.89 & 0.02 & 5.85 \\
\hline \multirow{5}{*}{ CL-A } & 400 & 4.34 & 0.6 & 4.11 & 8.38 & 0.70 & 3.49 \\
\hline & 800 & 11.69 & 0.99 & 6.40 & 24.78 & 0.72 & 4.59 \\
\hline & 1600 & 35.89 & 0.58 & 6.65 & 108.45 & 0.34 & 4.31 \\
\hline & 3200 & 149.23 & 0.12 & 5.85 & 411.79 & 0.13 & 4.82 \\
\hline & 6400 & 581.49 & 0.08 & 6.56 & 1573.80 & 0.06 & 4.79 \\
\hline
\end{tabular}

Table 2: Times and speedups for the different versions using both parameter sets and tested model sizes. The $\bar{t}(\mathrm{~s})$ column specifies the mean simulation time for each version and model size combination. The $s(\%)$ column shows the associated relative standard deviation, given by $100 \cdot s / \bar{t}(\mathbf{s})$, where $s$ is the sample standard deviation. The $S_{p}^{\text {Java }}$ column displays the speedups observed against the Java version.

Since parameter set 2 involves considerably more agents than the first, and thus entails more computationally expensive runs, API overheads seem to diminish and relative performance becomes more consistent.

From this comparison it becomes clear that using CPU-based OpenCL for implementing ABMs is a worthwhile endeavor. The speedups obtained against the parallel Java implementation offset the extra work required for developing a lower-level, CPUoptimized OpenCL implementation.

\subsection{Statistical comparison}

The OpenCL PPHPC implementation produces numerical equal results whether it is running on the Intel or AMD OpenCL runtimes. Thus, it is sufficient to compare the outputs of one or the other with the Java implementation, since they are the same.

The $p$-values obtained from the model-independent comparison of the Java and OpenCL PPHPC implementations for all combinations of parameter sets and model sizes are provided in Table 3. The $p$-values result from applying the MANOVA test on the first principal components which explain at least $90 \%$ of the respective output variance. The null hypothesis in this test is that the specified outputs from the two PPHPC versions are drawn from the same distribution. This comparison was performed with the micompr $\mathrm{R}$ package [22].

In a total of $60 p$-values ( 6 outputs, 2 parameter sets, 5 model sizes), 4 are below the 0.05 significance level, and none below 0.01 . The $p$-values do not appear to follow any trend or pattern, e.g., 


\begin{tabular}{rrrcccr}
\hline \multirow{2}{*}{ Size/set } & \multicolumn{7}{c}{ Outputs } \\
\cline { 2 - 7 } & $P^{s}$ & $P^{w}$ & $P^{c}$ & $\bar{E}^{s}$ & $\bar{E}^{w}$ & $\bar{C}$ \\
\hline $400 / 1$ & 0.062 & 0.285 & $\underline{0.029}$ & 0.241 & 0.446 & $\underline{0.030}$ \\
$800 / 1$ & 0.863 & 0.482 & 0.865 & $\underline{0.047}$ & 0.494 & 0.864 \\
$1600 / 1$ & 0.532 & 0.816 & 0.759 & 0.332 & 0.382 & 0.768 \\
$3200 / 1$ & 0.125 & 0.212 & 0.174 & 0.119 & 0.189 & 0.171 \\
$6400 / 1$ & 0.189 & 0.117 & 0.218 & $\underline{0.015}$ & 0.452 & 0.218 \\
\hline $400 / 2$ & 0.557 & 0.639 & 0.717 & 0.735 & 0.535 & 0.721 \\
$800 / 2$ & 0.522 & 0.560 & 0.558 & 0.289 & 0.724 & 0.559 \\
$1600 / 2$ & 0.623 & 0.822 & 0.787 & 0.297 & 0.655 & 0.786 \\
$3200 / 2$ & 0.153 & 0.567 & 0.715 & 0.830 & 0.654 & 0.715 \\
$6400 / 2$ & 0.996 & 0.989 & 0.990 & 0.997 & 0.882 & 0.990 \\
\hline
\end{tabular}

Table 3: $P$-values for the model-independent comparison of the Java and OpenCL PPHPC implementations (MANOVA test on the principal components which explain at least $90 \%$ of the respective output variance). Values lower than 0.05 are underlined.

smaller $p$-values do not seem to be associated with any particular output, parameter set or model size.

From these results, it is possible to conclude that the two model realizations seem to produce similar dynamic behavior. Thus, reimplementing PPHPC with OpenCL does not appear to have introduced any observable bias for the tested parameter sets.

\section{CONCLUSIONS AND FUTURE WORK}

In this paper we have compared a CPU-oriented OpenCL realization of the reference PPHPC ABM against a parallel Java implementation, which was already shown to be substantially faster than a serial NetLogo version. The OpenCL realization offered speedups up to $10 \times$ against the Java implementation on a 10-core hyper-threaded Xeon processor, while also providing fully reproducible simulations. Thus, it is possible to conclude that CPU variants of the OpenCL standard are appropriate for developing high-performance ABMs.

The presented OpenCL realization of the PPHPC model has the potential to be improved by using the nested parallelism capabilities introduced OpenCL 2.0. More specifically, these capabilities allow device-side kernel invocations, thus avoiding host-side row synchronization, shown in the inner for loops of Algorithm 3.

We are currently working on an agent-parallel GPU realization of PPHPC, which, at the time of writing requires a number of performance and simulation accuracy improvements [14].

\section{ACKNOWLEDGMENTS}

This work was funded by the Fundação para a Ciência e a Tecnologia project UID/EEA/50009/2013.

\section{REFERENCES}

[1] B. Aaby, K. Perumalla, and S. Seal. 2010. Efficient simulation of agent-based models on multi-GPU and multi-core clusters. In Proceedings of the 3rd International ICST Conference on Simulation Tools and Techniques. ICST (Institute for Computer Sciences, Social-Informatics and Telecommunications Engineering), $1-10$.
[2] S. Alberts, M. K. Keenan, R. M. D'Souza, and G. An. 2012. Data-parallel techniques for simulating a mega-scale agent-based model of systemic inflammatory response syndrome on graphics processing units. Simulation 88, 8 (2012), 895-907. DOI : http://dx.doi.org/10.1177/0037549711425180

[3] D. Chen, L. Wang, A. Zomaya, M. Dou, J. Chen, Z. Deng, and S. Hariri. 2015. Parallel Simulation of Complex Evacuation Scenarios with Adaptive Agent Models. IEEE Transactions on Parallel and Distributed Systems 26, 3 (March 2015), 847-857. DOI : http://dx.doi.org/10.1109/TPDS.2014.2311805

[4] S. Coakley, M. Gheorghe, M. Holcombe, S. Chin, D. Worth, and C. Greenough. 2012. Exploitation of High Performance Computing in the FLAME AgentBased Simulation Framework. In Proc. of the 14th Int. Conf. on High Performance Computing and Communications. IEEE, Liverpool, UK, 538-545. DOI : http://dx.doi.org/10.1109/HPCC.2012.79

[5] N. Collier and M. North. 2011. Repast HPC: A Platform for Large-Scale AgentBased Modeling. In Large-Scale Computing Techniques for Complex System Simulations, Werner Dubitzky, Krzysztof Kurowski, and Bernhard Schott (Eds.). John Wiley \& Sons, Inc., Chapter 5, 81-109. DOI : http://dx.doi.org/10.1002/ 9781118130506.ch5

[6] B. Cosenza. 2015. Behavioral Spherical Harmonics for Long-Range Agents' Interaction. Springer International Publishing, Cham, 392-404. DOI : http: //dx.doi.org/10.1007/978-3-319-27308-2_32

[7] B. Cosenza, G. Cordasco, R. De Chiara, and V. Scarano. 2011. Distributed load balancing for parallel agent-based simulations. In Parallel, Distributed and NetworkBased Processing (PDP), 2011 19th Euromicro International Conference on. IEEE, 62-69. DOI : http://dx.doi.org/10.1109/PDP.2011.22

[8] L. Dagum and R. Menon. 1998. OpenMP: an industry standard API for sharedmemory programming. IEEE Computational Science \& Engineering 5, 1 (1998), 46-55. DOI : http://dx.doi.org/10.1109/99.660313

[9] R. D’Souza, M. Lysenko, S. Marino, and D. Kirschner. 2009. Data-parallel algorithms for agent-based model simulation of tuberculosis on graphics processing units. In Proceedings of the 2009 Spring Simulation Multiconference (SpringSim '09). Society for Computer Simulation International, San Diego, CA, USA, 21:1-21:12.

[10] R. D'Souza, M. Lysenko, and K. Rahmani. 2007. SugarScape on steroids: simulating over a million agents at interactive rates. In Proc. of Agent 2007 Conf. Chicago, USA

[11] B. Edmonds and D. Hales. 2003. Replication, Replication and Replication: Some Hard Lessons from Model Alignment. Journal of Artificial Societies and Social Simulation 6, 4 (2003), 11. http://jasss.soc.surrey.ac.uk/6/4/11.html

[12] U. Erra, R. De Chiara, V. Scarano, and M. Tatafiore. 2004. Massive simulation using GPU of a distributed behavioral model of a flock with obstacle avoidance. In Proc. of vision, modeling and visualization.

[13] N. Fachada. 2008. Agent-based Simulation of the Immune System. Master's thesis. Instituto Superior Técnico, Universidade Técnica de Lisboa, Lisboa.

[14] N. Fachada. 2016. Agent-Based Modeling on High Performance Computing Architectures. Ph.D. Dissertation. DOI: http://dx.doi.org/10.13140/RG.2.2.11970.99523

[15] N. Fachada. 2016. cl_ops - a library of common OpenCL operations. GitHub. (2016). https://github.com/fakenmc/cl_ops

[16] N. Fachada, V. V. Lopes, R. C. Martins, and A. C. Rosa. 2015. Towards a standard model for research in agent-based modeling and simulation. Peerf Computer Science 1 (Nov. 2015), e36. DOI : http://dx.doi.org/10.7717/peerj-cs.36

[17] N. Fachada, V. V. Lopes, R. C. Martins, and A. C. Rosa. 2016. PerfAndPubTools Tools for Software Performance Analysis and Publishing of Results. Fournal of Open Research Software 4, 1 (may 2016). DOI : http://dx.doi.org/10.5334/jors.115

[18] N. Fachada, V. V. Lopes, R. C. Martins, and A. C. Rosa. 2017. cf4ocl: a C framework for OpenCL. Science of Computer Programming (March 2017). DOI : http://dx.doi. org $/ 10.1016 / j . s c i c o .2017 .03 .005$

[19] N. Fachada, V. V. Lopes, R. C. Martins, and A. C. Rosa. 2017. Model-independent comparison of simulation output. Simulation Modelling Practice and Theory 72 (March 2017), 131-149. DOI : http://dx.doi.org/10.1016/j.simpat.2016.12.013

[20] N. Fachada, V. V. Lopes, R. C. Martins, and A. C. Rosa. 2017. Parallelization Strategies for Spatial Agent-Based Models. International fournal of Parallel Programming 45, 3 (June 2017), 449-481. Issue 3. DOI : http://dx.doi.org/10.1007/ s10766-015-0399-9

[21] N. Fachada, V. V. Lopes, and A. Rosa. 2009. Simulating antigenic drift and shift in influenza A. In Proc. of the 2009 ACM symposium on Applied Computing (SAC '09). ACM, New York, NY, USA, 2093-2100. DOI : http://dx.doi.org/10.1145/1529282. 1529744

[22] N. Fachada, J. Rodrigues, V. V. Lopes, R. C. Martins, and A. C. Rosa. 2016. micompr: An R Package for Multivariate Independent Comparison of Observations. The $R$ fournal 8, 2 (Dec. 2016), 405-420. https://journal.r-project.org/archive/2016-2/ fachada-rodrigues-lopes-etal.pdf

[23] E. Gamma, R. Helm, R. Johnson, and J. Vlissides. 1994. Design patterns: elements of reusable object-oriented software. Pearson Education.

[24] M. J. Gibson, E. C. Keedwell, and D. A. Savić. 2015. An investigation of the efficient implementation of cellular automata on multi-core CPU and GPU hardware. 7 . Parallel and Distrib. Comput. 77 (2015), 11-25. DOI : http://dx.doi.org/10.1016/j. jpdc.2014.10.011 
[25] B. Göetz, T. Peierls, J. Bloch, J. Bowbeer, D. Holmes, and D. Lea. 2006. Java concurrency in practice. Addison-Wesley.

[26] M. E. Goldsby and C. M. Pancerella. 2013. Multithreaded agent-based simulation. In Proceedings of the 2013 Winter Simulation Conference: Simulation: Making Decisions in a Complex World (WSC '13). IEEE Press, Washington, D.C., USA $1581-1591$.

[27] Z. Gong, W. Tang, D. A. Bennett, and J.-C. Thill. 2013. Parallel agent-based simulation of individual-level spatial interactions within a multicore computing environment. International fournal of Geographical Information Science 27, 6 (2013), 1152-1170. DOI : http://dx.doi.org/10.1080/13658816.2012.741240

[28] S. Green. 2012. Particle simulation using CUDA. NVIDIA. (July 2012).

[29] V. Grimm, U. Berger, D. DeAngelis, J. Polhill, J. Giske, and S. Railsback. 2010. The ODD protocol: A review and first update. Ecological Modelling 221, 23 (2010), 2760-2768. DOI : http://dx.doi.org/10.1016/j.ecolmodel.2010.08.019

[30] L. Gulyás, A. Szabó, R. Legéndi, T. Máhr, R. Bocsi, and G. Kampis. 2011. Tools for large scale (distributed) agent-based computational experiments. In Proceedings of The Computational Social Science Society of the Americas.

[31] L. Gulyás, G. Szemes, G. Kampis, and W. de Back. 2009. A Modeler-friendly API for ABM partitioning. In Proceedings of the ASME 2009 International Design Engineering Technical Conferences \& Computers and Information in Engineering Conference. San Diego, CA, USA, 219-226.

[32] G. I. Hawe, G. Coates, D. T. Wilson, and R. S. Crouch. 2012. Agent-based Simulation for Large-scale Emergency Response: A Survey of Usage and Implementation. Comput. Surveys 45, 1 (Dec. 2012), 8:1-8:51. DOI : http://dx.doi.org/10.1145/ 2379776.2379784

[33] D. R. C. Hill, C. Mazel, J. Passerat-Palmbach, and M. K. Traore. 2013. Distribution of random streams for simulation practitioners. Concurrency and Computation Practice and Experience 25, 10 (2013), 1427-1442. DOI : http://dx.doi.org/10.1002/ cpe. 2942

[34] L. Howes. 2015. The OpenCL Specification, Version 2.1 (23 ${ }^{\text {rd }}$ ed.). Khronos OpenCL Working Group.

[35] A. Husselmann and K. Hawick. 2008. Spatial Agent-based Modelling and Simulations - A Review. Research Letters in the Information and Mathematical Sciences 7 (2008), 101-111.

[36] A. G. Isaac. 2011. The ABM Template Models: A Reformulation with Reference Implementations. Journal of Artificial Societies and Social Simulation 14, 2 (2011) 5. http://jasss.soc.surrey.ac.uk/14/2/5.html

[37] A. Jeannin-Girardon, P. Ballet, and V. Rodin. 2013. An Efficient Biomechanical Cell Model to Simulate Large Multi-cellular Tissue Morphogenesis: Application to Cell Sorting Simulation on GPU. Springer Berlin Heidelberg, Berlin, Heidelberg, 96-107. DOI: http://dx.doi.org/10.1007/978-3-642-45008-2_8

[38] M. Keenan, I. Komarov, R. M. D’Souza, and R. Riolo. 2012. Novel graphics processing unit-based parallel algorithms for understanding species diversity in forests. In Proceedings of the 2012 Symposium on High Performance Computing. Society for Computer Simulation International, 10

[39] K. Kofler, G. Davis, and S. Gesing. 2014. SAMPO: An Agent-based Mosquito Point Model in OpenCL. In Proceedings of the 2014 Symposium on Agent Directed Simulation (ADS '14). Society for Computer Simulation International, San Diego, CA, USA, Article 5, 10 pages. http://dl.acm.org/citation.cfm?id=2665049.2665054

[40] G. Laville, K. Mazouzi, C. Lang, N. Marilleau, B. Herrmann, and L. Philippe 2014. MCMAS: A Toolkit to Benefit from Many-Core Architecure in Agent-Based Simulation. Springer Berlin Heidelberg, Berlin, Heidelberg, 544-554. DOI : http //dx.doi.org/10.1007/978-3-642-54420-0_53

[41] D. E. Lenoski and W.-D. Weber. 1991. Scalable shared-memory multiprocessing. Elsevier.

[42] H. Lorek and M. Sonnenschein. 1995. Using parallel computers to simulate individual-oriented models in ecology: A case study. In Proceedings of the 1995 European Simulation Multiconference (ESM). 526-531.

[43] S. Luke, C. Cioffi-Revilla, L. Panait, K. Sullivan, and G. Balan. 2005. MASON A multiagent simulation environment. Simulation 81, 7 (2005), 517-527. DOI : http://dx.doi.org/10.1177/0037549705058073

[44] M. Lysenko and R. D'Souza. 2008. A framework for megascale agent based model simulations on graphics processing units. Fournal of Artificial Societies and Social Simulation 11, 4 (2008), 10. http://jasss.soc.surrey.ac.uk/11/4/10.html

[45] C. Macal and M. North. 2010. Tutorial on agent-based modelling and simulation. fournal of Simulation 4, 3 (2010), 151-162.

[46] M. Matsumoto and T. Nishimura. 1998. Mersenne twister: a 623-dimensionally equidistributed uniform pseudo-random number generator. ACM Transactions on Modeling and Computer Simulation 8, 1 (1998), 3-30. DOI : http://dx.doi.org/10. $1145 / 272991.272995$

[47] U. Merlone, M. Sonnessa, and P. Terna. 2008. Horizontal and Vertical Multiple Implementations in a Model of Industrial Districts. Fournal of Artificial Societie and Social Simulation 11, 2 (2008), 5. http://jasss.soc.surrey.ac.uk/11/2/5.html

[48] D. Moser, A. Riener, K. Zia, and A. Ferscha. 2011. Comparing Parallel Simulation of Social Agents using Cilk and OpenCL. In Distributed Simulation and Real Time Applications (DS-RT), 2011 IEEE/ACM 15th International Symposium on. IEEE, 88-97. DOI : http://dx.doi.org/10.1109/DS-RT.2011.12
[49] M. J. North, N. T. Collier, J. Ozik, E. R. Tatara, C. M. Macal, M. Bragen, and P. Sydelko. 2013. Complex adaptive systems modeling with Repast Simphony. Complex Adaptive Systems Modeling 1, 1 (2013), 1-26. DOI : http://dx.doi.org/10. 1186/2194-3206-1-3

[50] H. R. Parry and M. Bithell. 2012. Large Scale Agent-Based Modelling: A Review and Guidelines for Model Scaling. In Agent-Based Models of Geographical Systems, Alison J. Heppenstall, Andrew T. Crooks, Linda M. See, and Michael Batty (Eds.). Springer Netherlands, 271-308. DOI : http://dx.doi.org/10.1007/ 978-90-481-8927-4 14

[51] E. Passos, M. Joselli, M. Zamith, J. Rocha, A. Montenegro, E. Clua, A. Conci, and B. Feijó. 2008. Supermassive crowd simulation on GPU based on emergent behavior. In Proceedings of the VII Brazilian Symposium on Computer Games and Digital Entertainment. 81-86.

[52] K. Perumalla and B. Aaby. 2008. Data Parallel Execution Challenges and Runtime Performance of Agent Simulations on GPUs. In Proceedings of the 2008 Spring Simulation Multiconference. Society for Computer Simulation International, San Diego, CA, USA, 116-123

[53] K. S. Perumalla. 2010. Computational Spectrum of Agent Model Simulation. In Modeling Simulation and Optimization - Focus on Applications, Shkelzen Cakaj (Ed.). InTech, Chapter 12, 185-204.

[54] P. Richmond, D. Walker, S. Coakley, and D. Romano. 2010. High performance cellular level agent-based simulation with FLAME for the GPU. Briefings in Bioinformatics 11, 3 (2010), 334-347. DOI : http://dx.doi.org/10.1093/bib/bbp073

[55] M. Scheutz and P. Schermerhorn. 2006. Adaptive algorithms for the dynamic distribution and parallel execution of agent-based models. F. Parallel and Distrib. Comput. 66, 8 (2006), 1037-1051.

[56] E. Shook, S. Wang, and W. Tang. 2013. A communication-aware framework for parallel spatially explicit agent-based models. International fournal of Geographical Information Science 27, 11 (2013), 2160-2181. DOI : http://dx.doi.org/10.1080/ 13658816.2013 .771740

[57] J. E. Stone, D. Gohara, and G. Shi. 2010. OpenCL: A parallel programming standard for heterogeneous computing systems. Computing in Science \& Engineering 12, 3 (May 2010), 66-73. DOI : http://dx.doi.org/10.1109/MCSE.2010.69

[58] J. A. Stuart and J. D. Owens. 2011. Efficient Synchronization Primitives for GPUs. arXiv abs/1110.4623 (2011). http://arxiv.org/abs/1110.4623

[59] T. Takahashi and H. Mizuta. 2006. Efficient agent-based simulation framework for multi-node supercomputers. In Proceedings of the 38th conference on Winter simulation. Winter Simulation Conference, 919-925.

[60] W. Tang and D. Bennett. 2011. Parallel agent-based modeling of spatial opinion diffusion accelerated using graphics processing units. Ecological Modelling 222, 19 (2011), 3605-3615. DOI : http://dx.doi.org/10.1016/j.ecolmodel.2011.08.016

[61] W. Tang and M. Jia. 2014. Global sensitivity analysis of a large agent-based model of spatial opinion exchange: A heterogeneous multi-GPU acceleration approach. Annals of the Association of American Geographers 104, 3 (2014), 485-509. DOI : http://dx.doi.org/10.1080/00045608.2014.892342

[62] W. Tang and S. Wang. 2009. HPABM: A Hierarchical Parallel Simulation Framework for Spatially-explicit Agent-based Models. Transactions in GIS 13, 3 (2009), 315-333. DOI : http://dx.doi.org/10.1111/j.1467-9671.2009.01161.x

[63] The GNOME Project 2017. GLib Reference Manual. The GNOME Project. https: //developer.gnome.org/glib/ v2.44.0.

[64] D. B. Thomas. 2011. The MWC64X Random Number Generator. (2011). http: //cas.ee.ic.ac.uk/people/dt10/research/rngs-gpu-mwc64x.html

[65] G. Vigueras, J. M. Orduña, M. Lozano, J. M. Cecilia, and J. M. García. 2013. Accelerating collision detection for large-scale crowd simulation on multi-core and many-core architectures. International fournal of High Performance Computing Applications (Feb. 2013). DOI : http://dx.doi.org/10.1177/1094342013476119

[66] A. Voss, J.-Y. You, E. Yen, H.-Y. Chen, S. Lin, A. Turner, and J.-P. Lin. 2010. Scalable Social Simulation: Investigating Population-Scale Phenomena Using Commodity Computing. In e-Science (e-Science), 2010 IEEE Sixth International Conference on. IEEE, Brisbane, QLD, Australia, 1-8. DOI : http://dx.doi.org/10.1109/eScience. 2010.46

[67] J. Wang, N. Rubin, H. Wu, and S. Yalamanchili. 2013. Accelerating simulation of agent-based models on heterogeneous architectures. In Proceedings of the 6th Workshop on General Purpose Processor Using Graphics Processing Units (GPGPU6). ACM, Houston, Texas, USA, 108-119. DOI : http://dx.doi.org/10.1145/2458523. 2458534

[68] K. Wang and Z. Shen. 2012. A GPU based trafficparallel simulation module of artificial transportation systems. In Service Operations and Logistics, and Informatics (SOLI), 2012 IEEE International Conference on. IEEE, Suzhou, China, 160-165. DOI : http://dx.doi.org/10.1109/SOLI.2012.6273523

[69] U. Wilensky. 1999. NetLogo. (1999). http://ccl.northwestern.edu/netlogo/

[70] U. Wilensky and W. Rand. 2007. Making models match: replicating an agentbased model. Journal of Artificial Societies and Social Simulation 10, 4 (2007), 2. http://jasss.soc.surrey.ac.uk/10/4/2.html 